\title{
The sciatic nerve in human cadavers - high division or low formation?
}

\author{
A.A. Khan'1, M.A. Asari², M.A. Pasha ${ }^{3}$ \\ ${ }^{1}$ Department of Clinical and Neuroanatomy, Universiti Sains, Malaysia \\ ${ }^{2}$ Department of Neurohistology, Universiti Sains. Malaysia \\ ${ }^{3}$ Department of Surgery and Clinical Anatomy, Universiti Sains. Malaysia \\ [Received: 28 October 2015; Accepted: 13 November 2015]
}

\begin{abstract}
Variations of the sciatic nerve have been extensively studied in the past including its relationship with the piriformis muscle and associated clinical conditions like piriformis syndrome and sciatica. In the present study we noticed some interesting variations of the sciatic nerve, which were slightly different from the cases described earlier. In the previous studies most of the authors described the higher division of sciatic nerve and none of them discussed its formation. In this study we tried to look its formation from the sacral plexus and its divisions in the thigh. We noticed that in one cadaver the two components of the sciatic nerve originated directly from the sacral plexus and coursed down without merging in the thigh. Should this be called a higher division or non formation of the sciatic nerve? On the other hand in two other cadavers, the two divisions after emerging separately from the sacral plexus, united in the gluteal region and in the thigh respectively. Should we call this as higher division or low formation of the sciatic nerve? In two other cadavers the sciatic nerve emerged from the greater sciatic foramen below the piriformis and divided in the gluteal region itself. Ideally this should be called as higher division of sciatic nerve. (Folia Morphol 2016; 75, 3: 306-310)
\end{abstract}

Key words: sciatic nerve, common fibular nerve, tibial nerve, piriformis muscle, greater sciatic foramen

\section{INTRODUCTION}

The sciatic nerve (SN) is the largest branch of the sacral plexus. It has two components, the tibial nerve (TN) and common fibular nerves (CFN) enclosed in a common fascial sheath. In the majority of cases, SN leaves the pelvic cavity by passing through the greater sciatic foramen below the piriformis muscle and normally it divides into two separate trunks outside the pelvis, usually at the level of the upper angle of the popliteal fossa [22].

Variations in the level of division of SN are not uncommon phenomena. In most cases of high division, the SN separates after emerging from the greater sci- atic foramen but quite infrequently it separates within the pelvic cavity. The incidence of high division of SN, that is, the division of $\mathrm{SN}$ above its usual site varies considerably. For instance, the reported incidences of SN division before its exit in the gluteal region vary from $4.0 \%$ [23], to $20.9 \%$ [17] to $48.0 \%$ [7].

Regarding the relationship between SN to piriformis, various studies reported a variety of different anatomic relations between the $\mathrm{SN}$ or its terminal branches and the piriformis $[2,5,6,10,19]$. There are two important aspects with regard to SN variations, first, its position in relation to piriformis muscle and secondly, the location where it divides into two

Address for correspondence: Dr A.A. Khan, Department of Clinical and Neuroanatomy, Universiti Sains, Health Campus, USM Kubag Kerian, 16150, Kelantan, Malaysia, e-mail: aaijazk@gmail.com 
terminal trunks. In some cases SN may emerge above the piriformis or through the muscle. While in other variants its branches, CFN and TN may lie on either side of the muscle, or one branch either above or below or one branch passing through the piriformis (the most common variant). These varied relationships of the SN and its branches with the piriformis muscle, while passing through the greater sciatic foramen may result in non-discogenic sciatica or piriform syndrome [16, 20, 21]. The piriformis syndrome is a group of symptoms that include low back or buttock pain referred to the leg. It can be described as a nerve entrapment syndrome characterised by the entrapment of the $\mathrm{SN}$ by piriformis muscle hypertrophy, inflammation, or irritation [3, 15]. Further Güvençer et al. [7] stated that each anatomical variation may reflect a different and a case-specific clinical presentation. In addition, higher division of the SN may be the cause of an incomplete block of the SN during popliteal block anaesthesia [18].

While most studies describe the division of SN none have described its low formation. Noting the importance of high division or low formation of SN and the incidence of piriformis syndrome due to anomalous relationship between piriformis muscle and SN and its terminal branches, we undertook this study with the aim to highlight interesting cases of high division of $\mathrm{SN}$ and its relation with the piriformis muscle as well as low formation of SN. We hope our findings will be helpful to the clinician seeking to explain causes of sciatica and piriformis syndrome.

\section{MATERIALS AND METHODS}

We dissected 24 lower limbs of formalin fixed male cadavers available in the Department of Anatomy, Health Campus, Universiti Sains Malaysia to see the variations of the division of the SN. After careful dissection $\mathrm{SN}$ was exposed in the thigh and gluteal region. We noted the division of the $\mathrm{SN}$ in all the specimens. In specimens, who were having high division of $\mathrm{SN}$, we tried to define the exact location of the division. In three specimens, in whom CFN and TN nerves were coming separately through the greater sciatic foramen, we tried to dissect the sacral plexus in the pelvis to see the origin of these two branches from the sacral plexus. All the details were recorded and photographs were taken.

\section{RESULTS}

Out of 24 limbs dissected, in 6 limbs we found interesting variation of SN. In three limbs (No. 1, 2

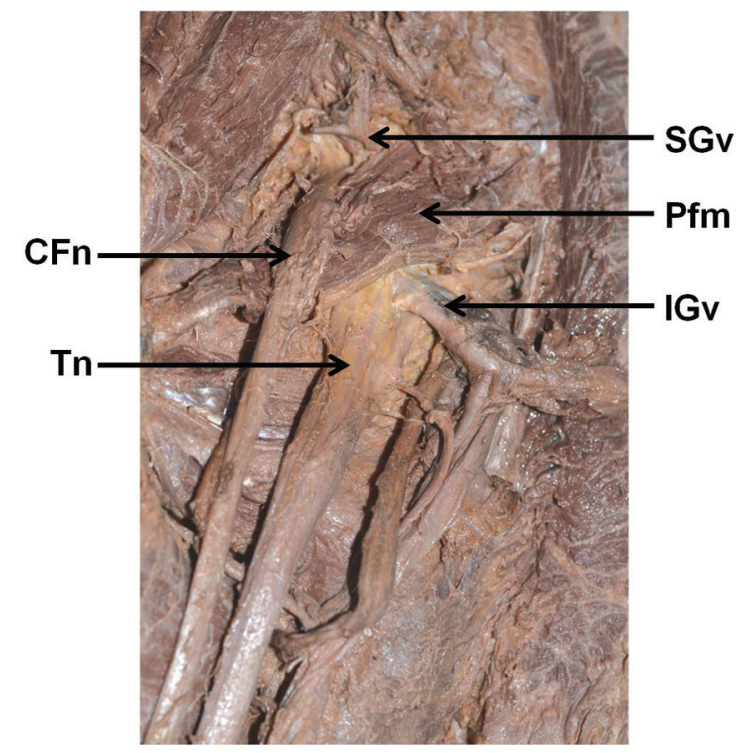

Figure 1. The left gluteal region after removing gluteus maximus; $\mathrm{CFn}$ - common fibular nerve; $\mathrm{Tn}$ - tibial nerve; SGv — superior gluteal vessels; IGv — inferior gluteal vessels; Pfm — piriformis muscle.

and 3), TN and CFN were seen emerging through the greater sciatic foramen separately. In specimen 4, 5 and 6 , SN was dividing below the piriformis in the thigh. In specimen 4 and 5 it was dividing high up in the thigh, while in specimen 6 , it was dividing quite low in the popliteal fossa and sural nerve and sural communicating nerve were arising from the CFN. In the other 18 limbs we did not find any such variations.

Out of 3 limbs with so called higher division of $\mathrm{SN}$, in case 1 (Fig. 1) CFN exited the pelvis above the piriformis (SP) and TN emerged below the piriformis (IP). Following sacral plexus exploration, we noticed that the lumbosacral trunk was coursing down and receiving contribution from first sacral primary ramus and then dividing into two roots just above the piriformis muscle; one root was continuing down through SP as CFN and the other root joined the TN just behind the piriformis muscle (Fig. 2). Thus, in this limb TN had formed quite low just before emerging through the greater sciatic foramen. By looking at this arrangement we surmise that $\mathrm{SN}$ is not formed at all in this limb and CFN and TN are direct branches from the sacral plexus. Interestingly in this specimen we also noticed that inferior gluteal vessels were passing through the TN and peroneal communicating nerve was arising in the middle of the thigh (Fig. 2).

In case 2 (Fig. 3), CFN was seen passing through the lower part of the piriformis muscle along with the inferior gluteal vessels and TN emerged through 


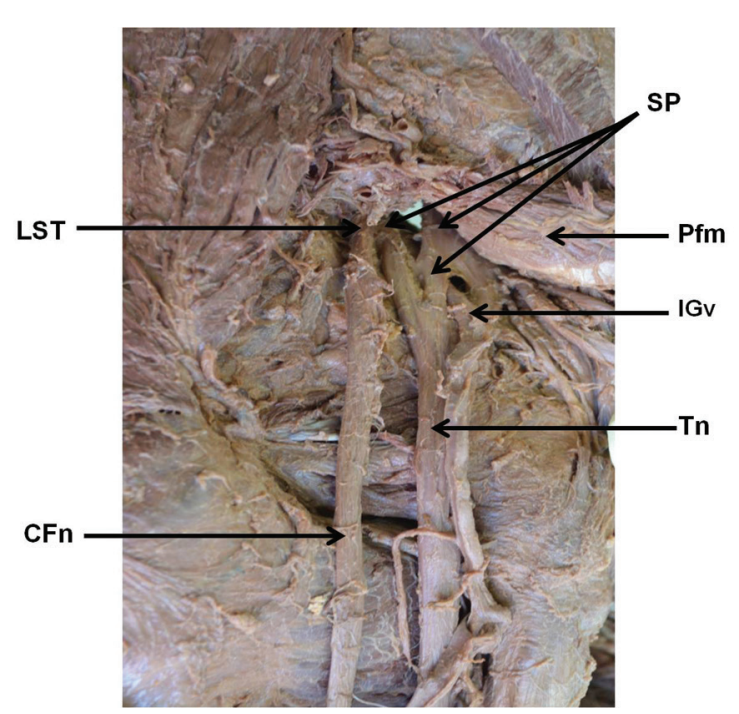

Figure 2. The left gluteal region showing the region of greater sciatic foramen after reflecting piriformis muscle; LST — lumbosacral trunk; $\mathrm{SP}$ - sacral plexus; $\mathrm{CFn}$ - common fibular nerve; $\mathrm{Tn}$ — tibial nerve; IGv — inferior gluteal vessels; Pfm — piriformis muscle.

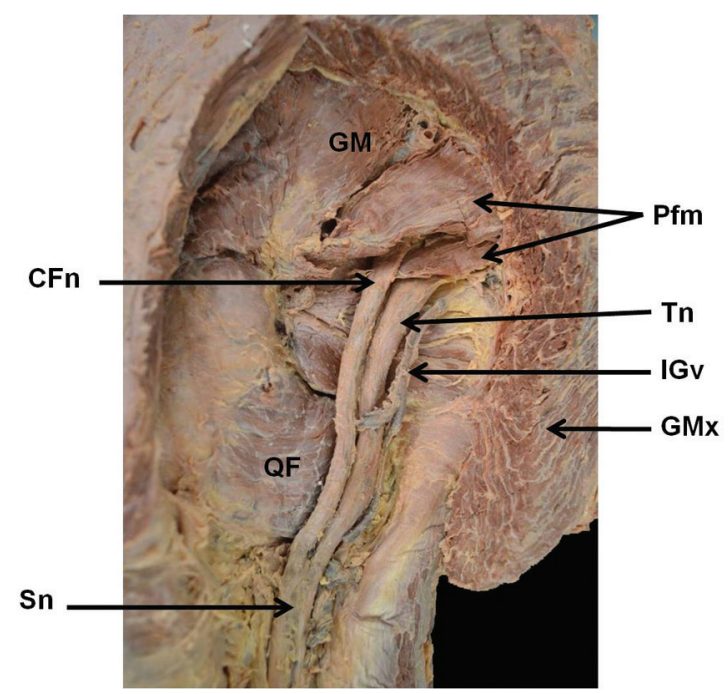

Figure 3. The left gluteal region after reflecting gluteus maximus muscle; $\mathrm{CFn}$ - common fibular nerve; $\mathrm{Tn}$ - tibial nerve; IGv inferior gluteal vessels; Pfm — piriformis muscle; GM — gluteus medius; $\mathrm{OF}$ - quadratus femoris muscle; $\mathrm{GMx}$ - gluteus maximus muscle; $\mathrm{Sn}$ - sciatic nerve.

the IP of the greater sciatic foramen. In the gluteal region both the nerves were running down close to each other and at the lower border of the quadratus femoris muscle these two divisions were seen to be enclosed in a common facial sheath and then pursue a normal course in the thigh as the SN.

In case 3 (Fig. 4), TN and CFN emerged separately from the greater sciatic foramen. CFN was passing

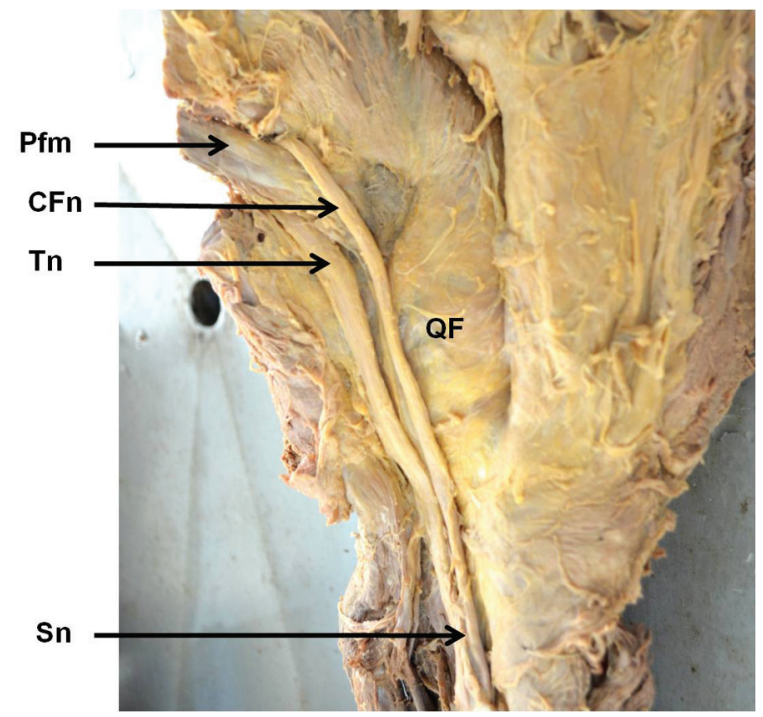

Figure 4. The right gluteal region after reflecting gluteus maximus muscle; $\mathrm{CFn}$ - common fibular nerve; $\mathrm{Tn}$ — tibial nerve; $\mathrm{Pfm}$ piriformis muscle; $\mathrm{OF}$ — quadratus femoris muscle; $\mathrm{Sn}$ - sciatic nerve.

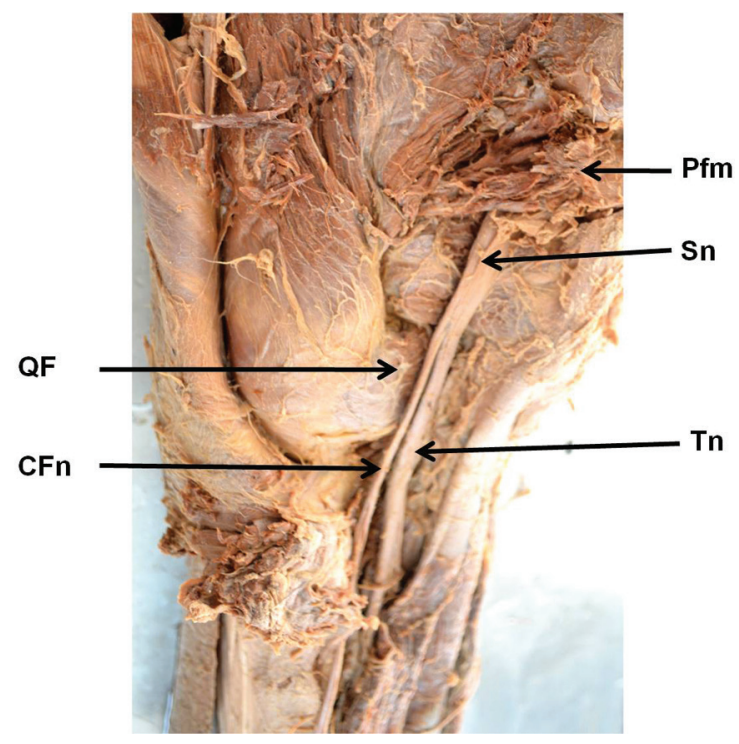

Figure 5. The left gluteal region after reflecting gluteus maximus muscle; CFn — common fibular nerve; $\mathrm{Tn}$ — tibial nerve; Pfm piriformis muscle; $\mathrm{OF}$ - quadratus femoris muscle; $\mathrm{Sn}$ - sciatic nerve.

above and TN had passed below the piriformis. Interestingly these two divisions united in the upper part of the thigh to form the SN.

In case 4 (Fig. 5), SN was seen dividing just at the upper border of the quadratus femoris muscle in the gluteal region and in case 5 (Fig. 6), it was dividing just after emerging through the greater sciatic foramen below the piriformis muscle. 


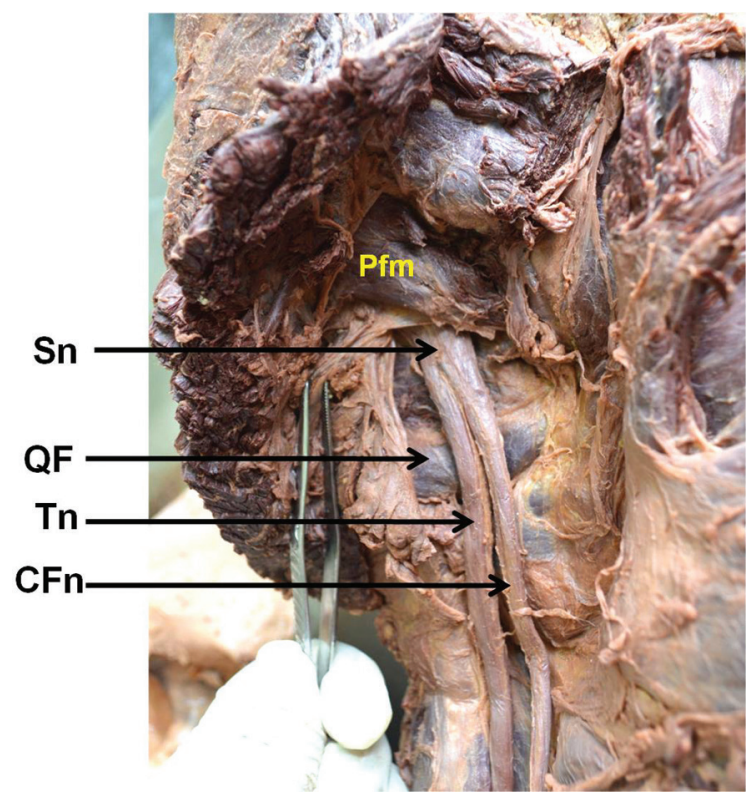

Figure 6. The right gluteal region after reflecting gluteus maximus muscle; CFn - common fibular nerve; $\mathrm{Tn}$ - tibial nerve; $\mathrm{Pfm}-$ piriformis muscle; $\mathrm{QF}$ - quadratus femoris muscle; $\mathrm{Sn}$ - sciatic nerve.

\section{DISCUSSION}

According to previous studies, higher division of $\mathrm{SN}$ in the thigh is not a very uncommon phenomenon but its division higher up in the pelvis or before emerging through the greater sciatic foramen and its anomalous relation with the piriformis is not so common.

Many previous studies on the varied relationship between the piriformis and $\mathrm{SN}$, have found variations in $15-30 \%$ of cases $[17,20]$.

Beaton and Anson [4] have classified these variations into six categories:

- Type 1: Undivided nerve below undivided piriformis muscle;

- Type 2: Divisions of nerve between and below undivided muscle;

- Type 3: Divisions above and below undivided muscle;

- Type 4: Undivided nerve between heads;

- Type 5: Divisions between and above heads;

- Type 6: Undivided nerve above undivided muscle. Out of these variations, type " 1 " is considered as normal relationship between the piriformis and the SN and type 2 to 6 are labelled as an abnormal relationship. The type 6 variation defined hypothetically by Beaton and Anson [4] was later reported by Ozaki et al. [14] and Sayson et al. [20]. Similarly Babinski et al. [2] and Mas et al. [11] reported a rare variation, in which CFN and TN emerged as separate branches through the IP but TN was passing anterior to the superior gemellus muscle. Though not reported by Beaton and Anson [4], Güvençer et al. [7] have referred to this variation as Beaton and Anson type 7 .

On the other, Machado et al. [10] reported three types of variation, including type 1 where the CFN penetrated the piriformis and the TN passed under the piriformis (16\%), type 2 where the CFN passed above the piriformis and the TN passed under the piriformis and type 3 where the SN penetrated the piriformis.

Pokorny et al. [17] customised the Beaton and Anson classification and stated that the type 1 variation, undivided nerve below undivided muscle, was seen in $79.1 \%$ of their specimens [16]. In our study we also noted that in 19 specimens the SN was dividing in the popliteal fossa.

In the present study we also found one specimen (case 2) with Type 2 variation in which CFN was penetrating the piriformis and TN was passing below the piriformis. Similar variant was reported in one case by Arifoğlu et al. [1], and by Kırıcı and Ozan [8]. Natsis et al. [13] dissected Caucasian cadavers (294 limbs) and only in 12 limbs (4.1\%) he found the CFN passed through and the TN below a double piriformis, while Güvençer et al. [7], found this variation in $14 \%$ of their cases. While two specimens ( 1 and 3 ) from our study appeared to be Beaton and Anson type 3 variation (Fig. 4), in which CFN was passing through the SP and the TN through the IP, these variations were reported by Ugrenovic et al. [23] in $1.5 \%$ of their cases. In our study, we noted that the two divisions were joining together and forming $\mathrm{SN}$ in the upper part of the thigh. No other study mentioned this type of SN formation. These cases cannot be called as a typical case of higher division of SN. In case 1 SN had not formed at all. The CFN and TN had originated separately from the sacral plexus and coursed down the thigh as two nerves. Other interesting feature which we found in this case is that the Inferior gluteal vessels were passing through the TN, which is not reported in any of the previous studies. Moreover, in this case first sacral nerve root was joining lumbosacral trunk quite low. Matejcik [12] also reported low level of connection between truncus lumbosacralis and first sacral nerve root in 10 cases. Contrarily in our cases 2 and 3, CFN and TN after emerging through the greater sciatic foramen as separate branches, joined together in the upper part of the thigh forming the SN. We suggest that this arrangement be termed as low formation of SN rather than a higher division of SN. 
We did not find Beaton and Anson type 4 variations as this variation is extremely rare and is reported only by Chen [5], and by Kosukegawa et al. [9].

Case 4 and 5 are typically called as higher division of the $\mathrm{SN}$, as in these cases $\mathrm{SN}$ was dividing after emerging from the IP of the greater sciatic foramen high up in the thigh. This type of variation is not described by Beaton and Anson [4], Prakash et al. [18] reported this type of division in three (2 male and 1 female) out of 86 (3.5\%) extremities, they studied. Further they also stated that neurological sign and symptoms may depend on the level of SN division.

\section{CONCLUSIONS}

In three limbs the TN and CFN components of the SN had emerged separately with varied relationship with the piriformis. Out of these, in two cases, these two components had united in the thigh to form the $\mathrm{SN}$, while in the third case they had a separate course in the thigh. We do not consider this as a higher division of the SN. On the other hand we believe they should be termed as either low formation, if the two components joined together after emerging through the pelvis or failure of formation of the $\mathrm{SN}$, if these two components do not join at all. In the present study only in two limbs the SN was dividing in the gluteal region and we appropriately consider these cases as high division of the $\mathrm{SN}$. It is hoped our conclusions from this study will add a new perspective to the formation and division of the $\mathrm{SN}$, and help the clinician to better manage patients with sciatica and piriformis syndrome.

\section{REFERENCES}

1. Arifoğlu Y, Sürücü HS, Sargon MF, Tanyeli E, Yazar F (1997) Double superior gemellus together with double piriformis and high division of the sciatic nerve. Surg Radiol Anat, 19: 407-408.

2. Babinski MA, Machado FA, Costa WS (2003) A rare variation in the high division of the sciatic nerve surrounding the superiorgemellus muscle. Eur J Morphol, 41: 41-42.

3. Barton PM (1991) Piriformis syndrome: a rational approach to management. Pain, 47: 345-352.

4. Beaton LE, Anson BJ (1937) The relation of the sciatic nerve and its subdivisions to the piriformis muscle. Anat Rec, 70: 1-5.

5. Chen WS (1994) Bipartite piriformis muscle: an unusual case of sciatic nerve entrapement. Pain, 58: 269-272.

6. Chiba S (1992) Multiple positional relationships of nerves arising from the sacral plexus to the piriformis muscle in humans. Kaibogaku Zasshi, 67: 691-724.
7. Güvençer M, lyem C, Akyer P, Tetik S, Naderi S (2009) Variations in the high division of the sciatic nerve and relationship between the sciatic nerve and the piriformis. Turkish Neurosurg, 19: 139-144.

8. Kırıc Y, Ozan H (1999) Double gluteus maximus muscle with associated variations in the gluteal region. Surg Radiol Anat, 21: 397-400.

9. Kosukegawa I, Yoshimoto M, Isogai S, Nonaka S, Yamashita T (2006) Piriformis syndrome resulting from a rare anatomic variation. Spine, 31: 664-666.

10. Machado FA, Babinski MA, Brasil FB, Favorito LA, AbiduFigureiedo M, Costa MG (2003) Anatomical variations between sciatic nerve and priform muscle during fetal period in human. Int J Morphol, 21: 29-35.

11. Mas N, Ozekşi P, Ozdemir B, Kapakin S, Sargon MF, Celik HH, Yener N (2003) A case of bilateral high division of the sciatic nerves, together with a unilateral unusual course of the tibial nerve. Neuroanatomy, 2: 13-15.

12. Matejcík V (2010) Anatomical variations of lumbosacral plexus. Surg Radiol Anat, 32: 409-414.

13. Natsis K, Totlis T, Konstantinidis GA, Paraskevas G, Piagkou M, Koebke J (2014) Anatomical variations between the sciatic nerve and the piriformis muscle: a contribution to surgical anatomy in piriformis syndrome. Surg Radiol Anat, 36: 273-280.

14. Ozaki S, Hamabe T, Muro T (1999) Piriformis syndrome resulting from an anomalous relationship between the sciatic nerve and piriformis muscle. Orthopedics, 22: 771-772.

15. Papadopoulos EC, Khan SN (2004) Piriformis syndrome and low back pain: a new classification and review of the literature. Orthop Clin Am, 35: 65-71.

16. Pecina M (1979) Contribution to the etiological explanation of the piriformis syndrome. Acta Anat (Basel), 105: 181-187.

17. Pokorny D, Jahoda D, Veigl D, Pinskerova V, Sonsa A (2006) Topographic variations of the relationship of the sciatic nerve and the piriformis muscle and its relevance to palsy after total hip arthroplasty. Surg Radiol Anat, 28: 88-91.

18. Prakash, Bhardwaj AK, Devi MN, Sridevi NS, Rao PK, Singh G (2010) Sciatic nerve division: a cadaver study in the Indian population and review of them literature. Singapore Med J, 51: 721.

19. Robinson DR (1997) Pyriformis syndrome. The relation to sciatic pain. Am J Surg,73: 355-380.

20. Sayson SC, Ducey JP, Maybrey JB, Wesley RL, Vermilion D (1994) Sciatic entrapment neuropathy associated with an anomalous piriformis muscle. Pain, 59: 149-152.

21. Smoll NR (ALFABET!!) (2010) Variations of the piriformis and sciatic nerve with clinical consequence: a review. Clin Anat, 23: 8-17.

22. Standring $S$ (2005) Gray's anatomy. The anatomical basis of clinicalpractice. 39 Ed. Elsevier Churchill Livingstone, Spain 1403, 1404, 1446.

23. Ugrenovic S, Jovanovic I, Krstic V, Stojanovic V, Vasovic L, Antic S, Pavlovic S (2005) The level of the sciatic nerve division and its relations to the piriform muscle. Vojnosanit Pregl, 62: 45-49. 